\title{
Terapia antimicrobiana endovenosa ambulatoria: Alternativa a la hospitalización en un servicio de urgencia pediátrico
}

\author{
Thelma Suau C., Cecilia Piñera M., Sergio Díaz W. y Esteban Troncoso R.
}

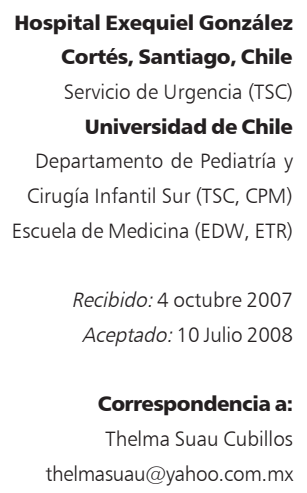

Hospital Exequiel González Servicio de Urgencia (TSC) Universidad de Chile Departamento de Pediatría y Cirugía Infantil Sur (TSC, CPM)

Recibido: 4 octubre 2007 Aceptado: 10 Julio 2008

Correspondencia a: thelmasuau@yahoo.com.mx

\section{Outpatient parenteral antimicrobial therapy: An alternative to hospitalization in a pediatric emergency departament}

Outpatient parenteral antimicrobial therapy (OPAT) was first introduced in 1973 as an alternative treatment. Since then, there have been numerous international case based studies including both children and adults with significant bacterial infections using this strategy. The protocol requires a careful screening and evaluation process of the patient. There must be no other motive for hospitalization other than the need for parenteral antibiotics. Objective: To describe the results of OPAT during a period of 26 months in emergency department of a Chilean pediatric public hospital. Results: During the study period 228,144 patients received medical care in the Emergency Department (ED) and 380 patients were admitted to the OPAT program after clinical evaluation and based on the socioeconomic and cultural conditions of their parents. The major indications of OPAT were skin and soft tissues infections (50\%) and pneumonia (28\%) respectively. $\beta$ lactamic antibiotics the were most commonly prescribed. Thirty eight patients $(10 \%)$ required hospitalization, mostly because of clinical deterioration. There were no deaths. Conclusion: In our public hospital OPAT for treatment of significant bacterial infections constitutes an efficient and safe alternative to hospitalization allowing the child to remain in his home.

Key words: Outpatient parenteral therapy, bacterial infections, antimicrobial, emergency department.

Palabras clave: Terapia endovenosa ambulatoria, infecciones bacterianas, antimicrobianos, servicio de urgencia.

\section{Introducción}

$\mathrm{L}$ a terapia antimicrobiana endovenosa ambulatoria (TAEA) surgió como una alternativa de tratamiento en los niños portadores de fibrosis quística. Desde los primeros reportes, que datan de $1973^{1}$, se han publicado numerosas experiencias internacionales en el manejo de distintas patologías infecciosas de la niñez y adultez, desde infecciones de piel y tejidos blandos, pasando por infecciones del tracto urinario (ITU), osteo-articulares, pulmonares, bacteriemias e incluso meningitis ${ }^{2-6}$.

Los estudios costo/beneficios de la TAEA fueron inicialmente realizados con el objetivo de disminuir los gastos hospitalarios en E.U.A. ${ }^{4}$. Sin embargo, con el tiempo se fue convirtiendo en una alternativa terapéutica para evitar la hospitalización, mantener al paciente inserto en su hogar y humanizar su atención ${ }^{7}$.

El uso de accesos endovenosos para pacientes ambulatorios tiene amplia distribución, y no sólo para la administración de antimicrobianos, sino también para alimentación parenteral de pacientes en su hogar y quimioterapia, entre otros ${ }^{8}$. Se han utilizado vías venosas periféricas, catéteres venosos centrales de inserción periférica (subcutáneo) y catéteres venosos centrales con reservorio subcutáneo ${ }^{9}$. En general, las complicaciones descritas asociadas a estos catéteres son infrecuentes ${ }^{8,9}$, aunque algunas series reportan incidencia de $50 \%$, pero de naturaleza leve $\mathrm{e}^{10}$.

La TAEA se plantea ante un paciente en quien se cumplen tres condiciones básicas: que esté cursando una infección bacteriana que requiere, al menos inicialmente, tratamiento antimicrobiano endovenoso (ev), que no exista otro motivo de hospitalización excepto la administración endovenosa del fármaco $\mathrm{y}$, que no se disponga de un tratamiento antibiótico oral equivalente en efectividad y seguridad ${ }^{9}$. Al seleccionar al paciente, el equipo médico debe verificar que se encuentre en buenas condiciones generales, no amerite cuidados hospitalarios y apruebe una evaluación de aspectos socioeconómicos y culturales, como la higiene del hogar y educación materna, entre otros. 
En el Hospital Exequiel González Cortés (HEGC), un establecimiento público terciario pediátrico, inserto en un sector socioeconómico medio-bajo de la Región Metropolitana chilena, se implementó la TAEA desde el año 1996, como consecuencia de la sobre-demanda asistencial y la escasez de camas para internar pacientes. Con el tiempo y mayor experiencia, se fue perfilando como un modelo alternativo de tratamiento, que permitió disminuir un número importante de hospitalizaciones?

El objetivo del presente estudio es describir la experiencia institucional en la implementación de la TAEA en la población infantil que consulta al Servicio de Urgencia (SU) del HEGC, revisar sus indicaciones médicas, duración del procedimiento en cada paciente, antimicrobianos utilizados, adherencia y complicaciones. A partir del diagnóstico actual podremos diseñar a futuro guías de práctica clínica basadas, tanto en la evidencia, como en la experiencia lograda.

\section{Pacientes y Métodos}

Estudio descriptivo, retrospectivo, de la experiencia en el SU del HEGC del programa de TAEA. Se revisó las fichas de enfermería de los pacientes ingresados al programa durante el período comprendido entre octubre de 2003 y diciembre de 2005. Se revisó además las fichas clínicas de aquellos pacientes que, luego de iniciar el protocolo, fueron internados en la misma institución.

Programa de TAEA. En los pacientes que consultaron al SU del HEGC y se les diagnosticó una patología que requirió tratamiento antimicrobiano ev, se seleccionó aquellos que podrían ser manejados de manera segura en forma ambulatoria, considerando para ello dos requisitos: condición médica (edad, estado general, patología, extensión de la misma y presencia de comorbilidad) y condición sociocultural/económica, evaluada mediante una encuesta que aplicaba la enfermera universitaria del SU (nivel de hacinamiento, distancia del hogar al hospital, nivel educacional de los padres y su ocupación, y elementos de saneamiento básico) (Anexo 1). Esta encuesta fue diseñada por el equipo de Salud al inicio del programa, asignándose puntaje cuyos límites fueron hechos por el equipo y que ha sido validada en la práctica. La encuesta tiene un puntaje entre 5 y 15 puntos, y sólo ingresaron a TAEA los pacientes con puntaje igual o mayor a 9 puntos. Aquellos con menos de 9 puntos eran hospitalizados. En aquellos niños incluidos en la TAEA, la enfermera instaló una vía venosa periférica de teflón, que permaneció instalada y administró las dosis del antimicrobiano, según la prescripción médica. Durante

\begin{tabular}{|c|c|}
\hline \multicolumn{2}{|l|}{ Anexo 1. Encuesta socio-económica } \\
\hline $\begin{array}{l}\text { 1. Hacinamiento } \\
\text { - Una persona por cama } \\
\text { - Cama compartida } \\
\text { * es requisito tener } 3 \text { puntos }\end{array}$ & $\begin{array}{l}3 \text { puntos } \\
1 \text { punto }\end{array}$ \\
\hline $\begin{array}{l}\text { 2. Distancia al hospital } \\
\text { - San Miguel, San Joaquín, P. Aguirre Cerda } \\
\text { - Lo Espejo, La Cisterna } \\
\text { - El Bosque, San Bernardo, La Pintana, otros }\end{array}$ & $\begin{array}{l}3 \text { puntos } \\
2 \text { puntos } \\
1 \text { punto }\end{array}$ \\
\hline $\begin{array}{l}\text { 3. Nivel educacional de los padres } \\
\text { - Universitario o técnico } \\
\text { - Educación media } \\
\text { - Educación básica o analfabeta }\end{array}$ & $\begin{array}{l}3 \text { puntos } \\
2 \text { puntos } \\
1 \text { punto }\end{array}$ \\
\hline $\begin{array}{l}\text { 4. Saneamiento básico } \\
\text { - Alcantarillado }+ \text { agua potable }+ \text { luz eléctrica } \\
\text { - Alcantarillado }+ \text { agua potable } \\
\text { - Sólo agua potable } \\
\text { * es requisito tener mínimo } 2 \text { puntos }\end{array}$ & $\begin{array}{l}3 \text { puntos } \\
2 \text { puntos } \\
1 \text { punto }\end{array}$ \\
\hline $\begin{array}{l}\text { 5. Ocupación de los padres } \\
\text { - Profesional o técnico } \\
\text { - Empleado u obrero } \\
\text { - Indigente o cesante }\end{array}$ & $\begin{array}{l}3 \text { puntos } \\
2 \text { puntos } \\
1 \text { punto } \\
\text { - puntos }\end{array}$ \\
\hline
\end{tabular}

la aplicación del protocolo cada paciente fue evaluado por médico, al menos una vez al día, quien decidió la mantención o suspensión del tratamiento endovenoso, cambio del tratamiento a vía oral u hospitalización.

\section{Resultados}

Datos demográficos. El HEGC tiene una población asignada de aproximadamente 117.337 pacientes (año 2005), con una demanda anual al SU de alrededor de 100.000 consultas, distribuidas en $68 \%$ consultas a pediatría, $20 \%$ a traumatología infantil y $12 \%$ a cirugía infantil. De un total de 228.144 consultas al SU durante el período comprendido entre octubre de 2003 y diciembre de 2005 (26 meses), 380 menores ingresaron a TAEA.

Nivel sociocultural de los hogares. El promedio de puntaje de la encuesta sociocultural/económica fue de 12 puntos. Sólo se ingresó a 5 niños con puntuación menor a 9 puntos.

El promedio de edad fue de 6,2 años (rango: 6 meses-15 años). Veintidós por ciento (n: 83) eran pacientes bajo 2 años de edad (Figura 1). La distribución por sexo fue de $57 \%$ varones y $43 \%$ niñas.

Distribución por comunas. 151 pacientes (40\%) residían en las comunas de San Miguel, San Joaquín y Pedro Aguirre Cerda, las más cercanas al hospital; 105 


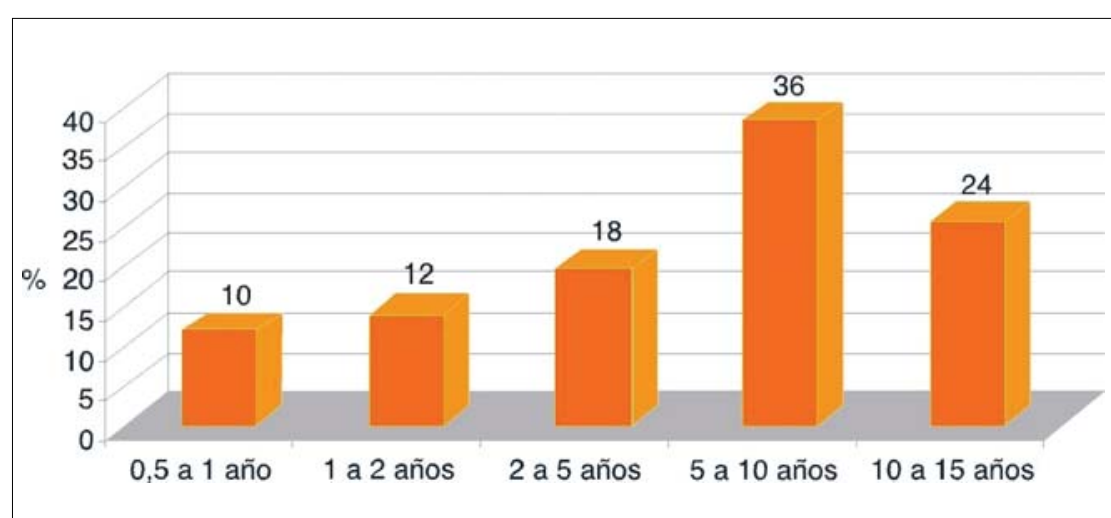

Figura 1. Distribución etaria de los pacientes que recibieron TAEA (n: 380). Hospital Exequiel González Cortés, 2003-2005.

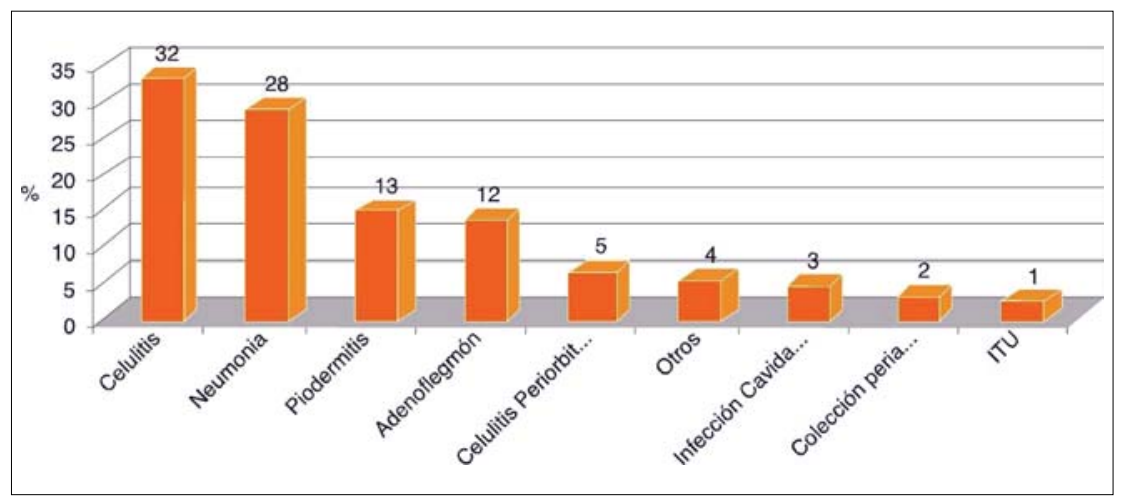

Figura 2. Distribución por diagnósticos. Pacientes en terapia antimicrobiana endovenosa ambulatoria (n: 380). Hospital Exequiel González Cortés, 2003-2005.

niños (28\%) en las comunas de Lo Espejo y La Cisterna; y 120 niños (32\%) en las comunas de El Bosque, San Bernardo y La Pintana, las más distantes del hospital.

Distribución por patologías. Las infecciones de piel y tejidos blandos representaron $50 \%$ y las neumonías $28 \%$ del total (Figura 2). Hubo sólo dos casos de infección urinaria. Se indicó TAEA en 17 pacientes $(5 \%)$ con celulitis periorbitaria pre-septal.

Indicación de la TAEA. En las infecciones de piel y tejidos blandos y en las neumonías, no pudimos obtener el fundamento de la indicación de ingreso a TAEA por falta de registro de este antecedente en la ficha de atención. Algunas de las causas consignadas fueron: mala tolerancia a antimicrobianos por vía oral, baja adherencia a tratamiento y grado de extensión de la lesión.

Prescripción de antimicrobianos. En general se utilizó penicilina $\mathrm{G}$ sódica para el tratamiento de neumonías y cloxacilina, en forma aislada o en esquema biasociado con penicilina $\mathrm{G}$ sódica $\mathrm{u}$ otro antimicrobiano, para las infecciones de piel y tejidos blandos. De este modo, los antimicrobianos más indicados fueron cloxacilina en 188 pacientes $(49,5 \%)$, seguido por penicilina en 135 pacientes $(35,5 \%)$, y esquema bi-asociado de cloxacilina + penicilina u otro antimicrobiano en $10 \%$ de los casos (38 pacientes). Otros antimicrobianos utilizados fueron clindamicina, amoxicilina/ácido clavulánico, ceftriaxona, gentamicina y lincomicina. En $99 \%$ de los casos en que utilizó penicilina y/o cloxacilina la dosificación fue cada 8 horas.

Duración de la TAEA. En promedio, el tratamiento endovenoso se mantuvo por 2,7 días, (rango: 1-7 días). El número de dosis por paciente fue de 5,5 en promedio, (rango: 1-19 dosis).

Complicaciones del procedimiento. Hubo un subregistro en nuestra serie, ya que el protocolo de atención de los pacientes no contemplaba el registro de complicaciones leves, y quedó al criterio de la enfermera hacerlo. Se consignó complicaciones como flebitis y extravasación de la vía. No se describieron reacciones adversas a los medicamentos.

Resolución del procedimiento. El 90\% de los pacientes fue dado de alta a su hogar para continuar el tratamiento antimicrobiano vía oral, según correspondía. Treinta y ocho (10\%) pacientes ingresados al protocolo fueron internados, en el mismo establecimiento o fueron derivados a otro centro. Sólo 3 pacientes (menos del 1\%) abandonaron la TAEA. Del total que requirió hospitalización en segunda instancia, sólo se obtuvo información de los hospitalizados en el HEGC (17 en total). De ellos, 12 presentaban infección de tejidos blandos (celulitis, abscesos y adeno-flegmones) y el motivo de hospitalización fue la progresión de la lesión y/o la persistencia de fiebre. Dos de ellos requirieron drenaje quirúrgico: uno portador de un absceso maxilar y un menor de 5 años con un absceso perianal. Cuatro pacientes tenían neumonía, dos de ellos se hospitalizaron por evolucionar con insuficiencia respiratoria parcial, uno por persistir febril, y el cuarto por evolución tórpida con extensión de la consolidación en la radiografía de tórax. Una menor de un año que se internó por evolucionar con insuficiencia respiratoria requirió manejo en la Unidad de Cuidados Intermedios y apoyo con ventilación mecánica no invasora durante 48 horas. El promedio de días de hospitalización para los 38 pacientes fue de tres días (rango: 1-7 días). Ningún paciente falleció.

\section{Discusión}

La TAEA constituye una alternativa a la hospitalización frente a niños que cursan con infecciones bacterianas que requieren inicialmente de tratamiento endovenoso pero que, por encontrarse en buenas con- 
diciones generales, no requieren cuidado hospitalario salvo la administración del fármaco. Es de fundamental importancia que esta modalidad sea segura, con mínimo riesgo de complicaciones. Por esto los programas de TAEA deben ser multidisciplinarios, con la participación del médico, equipo de enfermería, de farmacia e idealmente, del servicio social. Al considerar a un niño como candidato a TAEA, el equipo médico debe cerciorarse no sólo de las aceptables condiciones de salud (edad, extensión de la infección, co-morbilidad), sino también de las condiciones en el hogar y de la calificación de su apoderada (o), ya que son los padres o tutores quienes deberán vigilar su evolución en el hogar del niño.

Idealmente se debiera realizar una encuesta domiciliaria y evaluación de las condiciones del hogar por parte de una enfermera o asistente social, como se realiza en otros países ${ }^{11}$. Cabe señalar que en los países desarrollados es la madre quien administra el fármaco en el hogar ${ }^{12}$, a diferencia de lo que ocurre en nuestro medio, en que el paciente debe acudir diariamente al servicio de urgencia para que una enfermera le administre el medicamento.

En la literatura médica norteamericana se han diseñado guías para la TAEA ${ }^{11,13}$ y promete ser una modalidad segura que en el tiempo se va a ir potenciando. En Chile, tenemos realidades distintas; los pacientes asignados a los hospitales públicos generalmente cuentan con menos recursos y con un nivel educacional inferior. Por esto creemos que la responsabilidad de administrar el antimicrobiano endovenoso, aún debe ser responsabilidad del equipo de salud (enfermera); en nuestro medio, estos pacientes requieren de un seguimiento y monitoreo mucho más cercano.

Una gran ventaja de este modelo de atención es que permite optimizar los recursos al "ahorrar" camas, llevando al nivel ambulatorio situaciones que tradicionalmente se manejaban dentro del hospital, de un modo seguro y eficiente. Así lo pudimos comprobar en esta casuística, en que se logró evitar 380 potenciales hospitalizaciones, en un período de tiempo de 26 meses. Sería interesante, a futuro, estimar la reducción en costos que esto significa. Por otro lado, manteniendo al margen los temas de costos y eficiencia, esta modalidad permite acercar la salud a las personas, manteniendo al niño inserto en su hogar y junto a su madre, en definitiva, humanizando su atención.

La distribución por patología encontrada en nuestro estudio difiere de la descrita en otras series; en nuestro caso $50 \%$ de los pacientes padecían de una patología infecciosa de piel y tejidos blandos, a diferencia de lo comunicado en la literatura científica internacional en que predominas las infecciones osteo$\operatorname{articulares}^{6,10,14}$. Mención especial hacemos de la fre- cuencia con que las celulitis periorbitarias fueron manejadas en forma ambulatoria, incluso en niños pequeños; esta condición, usualmente es atendida en el medio intra-hospitalario por las posibles complicaciones médicas de esta patología.

Lamentablemente, la falta de registro en el dato de atención de urgencia no nos permitió establecer la indicación de ingreso a TAEA en pacientes con neumonías e infección de tejidos blandos.

Otro punto a destacar es los antimicrobianos utilizados, difiriendo enormemente de las experiencias en países desarrollados, donde las cefalosporinas de $3^{\circ}$ generación, vancomicina y aminoglucósidos son utilizadas con mayor frecuencia ${ }^{6,14}$. Al respecto, cabe señalar que en nuestra experiencia las patologías más frecuentes fueron infecciones de piel y tejidos blandos con el consiguiente uso de penicilina $G$ sódica $y$ cloxacilina. Una autocrítica a nuestra experiencia es que se indicó principalmente antimicrobianos de vida media corta, requiriendo su administración varias veces al día. Esto implica no sólo una mayor probabilidad de complicaciones relacionadas con el catéter y mayor utilización de recurso humano, sino también un mayor costo para sus apoderados quienes debieron traer diariamente, varias veces, al hospital al paciente para la administración del fármaco. En el rediseño de un protocolo de TAEA para infecciones bacterianas deben seleccionarse antimicrobianos que requieran sólo una administración diaria.

En cuanto a las complicaciones de la vía de acceso hubo un sub-registro que no permitió evaluar este aspecto de la TAEA.

La tasa de hospitalización en segunda instancia encontrada en nuestro trabajo $(10 \%)$ fue baja en relación a la de otras series que comunican entre 10 y $26 \%$ como tasa de hospitalización en estas condiciones ${ }^{10}$. Al respecto, hay que considerar que los cursos de tratamiento antimicrobiano de las publicaciones extranjeras eran prolongados, más de dos semanas, en comparación al promedio de 2,7 días experimentado en nuestra serie.

Conclusiones. Esta revisión y análisis nos ha permitido identificar nuestras falencias, para que en un futuro próximo diseñemos protocolos basados en la evidencia y en nuestra experiencia clínica, llamando a participar en su diseño a distintos especialistas (pediatra, infectólogo, médico especialista en medicina de urgencia, enfermera, asistente social). De este modo, podremos optimizar un protocolo de TAEA como una alternativa más segura y eficiente, y eventualmente incorporar otras patologías a esta modalidad de tratamiento.

Agradecimientos. A Ana M. Chávez, Lilian Roa, José Luis Zúñiga, Trinidad Bascuñán y Magdalena Errázuriz. 


\section{Resumen}

La terapia antimicrobiana endovenosa ambulatoria (TAEA) surgió como una alternativa de tratamiento en 1973, publicándose numerosas experiencias internacionales en niños y adultos para diversas infecciones bacterianas de importancia mayor. Requiere seleccionar a cada paciente considerando que no hay otro motivo de hospitalización, salvo la administración del fármaco. Objetivo: Sistematizar la experiencia de TAEA efectuada durante 26 meses en el servicio de urgencia (SU) de un hospital pediátrico público de Chile. Resultados: De un total de 228.144 pacientes consultantes al
SU en el período elegido, un total de 380 pacientes ingresaron al programa de TAEA luego de una evaluación clínica y de las condiciones socioeconómicas y culturales de sus apoderados. La principal indicación de TAEA fueron las infecciones de piel y tejidos blandos (50\%), seguida de neumonía (28\%), utilizándose en su mayoría $\beta$-lactámicos solos o asociados. Diez por ciento de los pacientes requirió hospitalización, en su mayoría por deterioro de su condición clínica. Ninguno falleció. Conclusión: En nuestro medio, la TAEA es una alternativa eficiente y segura a la hospitalización, que permite tratar pacientes pediátricos con infecciones bacterianas mayores en su entorno familiar.

\section{Referencias}

1.- Rucker R W, Harrison G M. Outpatient intravenous medications in the management of cystic fibrosis. Pediatrics 1974; 54: 35860 .

2.- Maraqa N F, Gómez M, Rathore M. Outpatient parenteral antimicrobial therapy in osteoarticular infections in children. $\mathrm{J}$ Pediatr Orthop 2002; 22: 506-10.

3.- Balinsky W, Nesbitt S. Cost-effectiveness of outpatient parenteral antibiotics: a review of the literature. Am J Med 1989; 87: 301-5.

4.- Hindes R, Winckler C, Kane P, Kunkel M. Outpatient intravenous antibiotic therapy in Medicare patients: cost-savings analysis. Infect Dis Clin Pract 1995; 4: 211-7.

5.- Milkovich G. Benefits of outpatient parenteral antibiotic therapy: to the individual, the institution, third party payers and society. Int J Antimicrob Agents 1995; 5: $27-31$

6.- Graninger W, Wenisch C, Wiesinger E,
Menschik M, Karimi J, Prsterl E. Experience with a physician-directed, clinicbased program for outpatient parenteral antibiotic therapy in the USA. Eur J Clin Microbiol Infect Dis 1995; 14: 655-61.

7.- Aravena A, Aravena E, Caro U, Escalona J, Morales P, Muñoz D. Tratamiento endovenoso ambulatorio en la Unidad de Emergencia: una nueva modalidad de atención. Publicación Proyecto FrancoChileno de apoyo a la gestión hospitalaria. MINSAL. 1997.

8.- Arnett A M, Fleisher G R. Insertion of long lines in the pediatric emergency department. Pediatr Emerg Care 1999, 15: 318-21.

9.- Gilbert D, Dworkin R, Raber S, Pharm D, Leggett J. Outpatient parenteral antimicrobial-drug therapy. N Engl J Med 1997; 337: 829-38.

10.- Gómez M, Maraqa N, Álvarez A, Rathore M. Complications of outpatient parenteral antibiotic therapy in childhood. Pediatr Infect Dis J 2001; 20: 541-3.
11.- Tice A, Rehm S, Dalovisio J, Bradley J, Martinelli L, Gram D, et al. Practice guidelines for outpatient parenteral antimicrobial therapy. IDSA guidelines. Clin Infect Dis 2004; 38: 1651-71.

12.- Matthews P C, Conlon C P, Berendt A R, Kayley J, Jefferies L, Atkins BL. Outpatient parenteral antimicrobial therapy (OPAT): is it safe for selected patients to selfadminister at home? A retrospective analysis of a large cohort over 13 years. J Antimicrob Chemother 2007; 60: 356-62.

13.- Andrews M M, von Reyn C F. Patient selection criteria and management guidelines for outpatient parenteral antibiotic therapy for native infective endocarditis. Clin Infect Dis 2001; 33: 203-9.

14.- Chary A, Tice A, Martinelli L, Liedtke L, Plantenga M, Strausbaugh L. Experience of infectious disease consultans with parenteral antimicrobial therapy: results of an emerging infections network survey. Clin Infect Dis 2006; 43: 1290-5. 